\title{
Monopolistic Competition and New Products: A Conjectural Equilibrium Approach
}

\author{
Francesco Bogliacino*and Giorgio Rampa ${ }^{\dagger}$
}

August 25, 2008

\begin{abstract}
In this paper we generalize the models of diffusion of new products in a multifirms, heterogeneous and interacting agents environment.

We discuss the possibility of product failures, the set of equilibria, their stability and we close with some hints over welfare properties.

An important Note: this version is something in the middle between an extended abstract and a paper, it is thus incomplete. It has been written as a proposal for the WEHIA conference.

Keywords: Product Diffusion, Risk Aversion, Lock-in, Monopolistic Competition, Multiple Equilibria.

JEL Classification System: L15, D81, O33
\end{abstract}

\footnotetext{
${ }^{*}$ Universidad EAFIT, Departamento de Economía, and RISE Group, Carrera 49, 7 Sur 50, 05001000, Medellin (Colombia). E-mail: francesco.bogliacino@gmail.com

${ }^{\dagger}$ Dipartimento di Diritto Privato, Internazionale e Commerciale "G.L.M. CASAREGI" - Sezione Economica, Via Balbi, 22 - 16126 Genova - tel. 0102099891 - fax 0102095720, e-mail: g.rampa@unige.it
} 


\section{Introduction}

The analysis of the processes of diffusion is very important under at least two different perspectives. First of all, scholars usually talk about new products, but it is very simple to generalize conclusions to the adoption of new technologies, of new behaviours, and of new strategies (in the game theoretic sense) enlarging dramatically the focus. Secondly, it is multi-disciplinar in essence: the literature that studied the problem spans from management to sociology, from psychology to the multiple economic approaches.

The literature discussed both the conditions that favour or hamper the diffusion, eventually bringing it to failure or success; and the speed of diffusion, looking at the fundamentals of the potential patterns and in particular the possibility of an epidemiologic-like S-shaped curve.

We think that there exists some necessary building blocks. The first keywords is uncertainty: the novelty of goods (or ideas, tecnologies, behaviours etc.) implies that agents choose under some forms of conjecture over the unknows parameters, as in standard bayesian approach (Jensen 1982, Feder and O'Mara 1982, Tsur et al. 1990, Chatterjee and Eliashberg 1990, Young 2006). The second one is heterogeneity: the individual models should be different, since they sum up conjectures, previous learning, a priori ideas (Abrahamson and Rosenkopf, 1993; Cowan and Jonard 2003 and 2004; Lopez Pintado and Watts, 2006). The third one is interaction: the learning of agents (from the past and from other agents) shapes the overall process, making it path dependent, allowing for non deterministic results (lock in) and finally culminating in multiple equilibria (see Arthur 1994, Amable 1992, Agliardi 1998, Aoki and Yoshikawa 2002, Young 2007).

In Bogliacino and Rampa 2008, we developed a setup, based on bayesian techniques, which includes risk aversion and a demand-and-supply interaction: the former is important because in the context of individual models, the emergence of information is likely to play a role in shaping the confidence of agents and this can be captured through the use of individual precisions; the latter allows to free the analysis from the one sided approach of all the literature, and to focus not just on consumers' uncertainty over quality, since it is also natural to assume firms' uncertainty over their own demand.

In this paper we generalize those results analyzing multiple goods together, abandoning the monopoly and moving towards monopolistic competition. Moreover, we still provide purely analytical results, characterizing the full set of equilibria, without relying on simple simulations exercises, which in the end gives only partial understanding of the overall process.

The paper proceeds as follows: Section 2 describes the demand side, Section 3 the supply one, Section 4 presents and discusses the main results, Section 5 concludes. 


\section{The Consumers}

The individual consumer maximizes her utility choosing the level of consumption of each one of $n$ new goods over whose qualities she is uncertain. Following standard bayesian setting, we assume consumers to be endowed with a prior over the quality, defined by two hyperparameters $\mu_{j, t}$ and $\tau_{i, t}$, respectively a mean and a precision (the inverse of the variance in this language, see DeGroot, 1970) that evolve through time being updated using Bayes' rule. We assume also additively separable preferences. From now on $j$ stands for the consumer, going from one to $M, i$ stands for product, going from 1 to $n$ and finally $t$ is time, ranging discretely from zero to infinite.

We can represent consumer's problem in the following way:

$$
\max _{\left\{x_{i}\right\}_{i=1}^{n}} E\left[U\left(x_{i, j}, \lambda_{i}\right) \mid \mu_{j, i, t}, \tau_{i, j, t}\right]=E\left[\sum_{i=1}^{n} u\left(x_{j, i}\right) f\left(\lambda_{i}\right) \mid \mu_{j, i, t}, \tau_{j, i, t}\right]
$$

such that

$$
\sum_{i=1}^{n} p_{i} x_{j, i} \leq w
$$

where $w$ is the income endowment, which can be equal through them.

The $f(\cdot)$ is the way in which quality of each good impacts over the choice. In particular, as in the one good framework of Bogliacino and Rampa 2008, we assume that they satisfy:

$$
\partial^{2} U /\left(\partial x_{j, i} \partial \lambda_{i}\right)>0
$$

meaning that the consumer wishes to purchase more if quality is higher, for given price; and

$$
\partial^{3} U /\left(\partial x_{j, i} \partial \lambda_{i}^{2}\right)<0
$$

i.e. the consumers are risk averse in quality: this suggests that a higher variance of quality tends to depress (expected) marginal utility and hence consumption, for given price. In Bogliacino and Rampa (2008) $u(\cdot)=(\cdot)^{\delta}$ and $f\left(\lambda_{i}\right)=A-\exp \left(-\lambda_{i}\right)$, with $\lambda_{i} \sim N\left(\mu_{i}, r\right)$, where $\mu_{i}$ is unknown and $r$ known. The individual prior is also normal, and allows us to use the properties of the conjugate family. The advantage of the specification of the $f(\cdot)$ is threefold: it satisfies the two conditions (2.1) and (2.2) above and allows us to pass through the expected value, using the fact that because of the normality and the exponential, the $f(\cdot)$ is log-normal; finally, whenever

$$
A<\exp \left(-\lambda_{i}\right)
$$

it should be meant that the consumer will not purchase any positive quantity of the good. This last property is useful to check the effects of noisy quality signals to the consumer, eventually addressing the possibility of lock-in: i.e. the failure of a diffusion of a "good" product. 
The quality of the good should be deemed either as a constant, such that through times the consumers learn and converge to the real value, with the interest standing in modeling the diffusion curve (e.g. to microfundate an epidemiologic or concave pattern), or as a random variable, affected by production or delivering process, such that the eventuality of a noisy signal can impact on the system.

A note on the timing: the consumer makes her choice using the all the information available, which is captured trough her posterior. Of course all the new information refers to the choices at $t-1$ and the hyperparameters relevant for the choice at $t$ are $\mu_{j, t-1}$ and $\tau_{j, t-1}$.

Standard maximization implies the following individual demand curve:

$$
x_{i, j, t}=\frac{p_{i}^{1 /(\delta-1)}\left(A-\exp \left(-\mu_{j, t-1}+\frac{\tau_{j, t-1}^{-1}+r^{-1}}{2}\right)\right)^{1 /(1-\delta)}}{\sum_{i} p_{i}^{\delta /(\delta-1)}\left(A-\exp \left(-\mu_{j, t-1}+\frac{\tau_{j, t-1}^{-1}+r^{-1}}{2}\right)\right)^{1 /(1-\delta)}} y
$$

and total demand for the good is just the summation over the $j$ index. The interpretation is straightforward: each consumer purchase a share of its total expenditure which dependes on the price quality ratio over the average of the bundle of goods.

Using the properties of conjugate families (DeGroot 1970) the posterior parameters for a normal-normal couple (being respectively the likelihood and the prior) are pretty simple to calculate:

$$
\mu_{i, t}=\frac{\tau_{i, t-1} \mu_{i, t-1}+r M_{t} \bar{\lambda}_{t}}{\tau_{i, t-1}+r M_{t}} \quad \text { and } \quad \tau_{i, t}=\tau_{i, t-1}+r M_{t}
$$

the above equation simply tells us that consumers average their own ideas and the sample mean of quality from the new observations (the new purchased by consumers, assuming that they publicly announce the quality observed), the weight being the relative precisions of the two measures. Moreover, through time the individual precision grows linearly: as one can imagine, given the assumptions over the quality-risk-aversion, this raise through time the demand, for a simple informational effect.

\section{The Firms}

The firms interact in monopolistic competition, each producing at a marginal cost equal to $c_{i}$. Each firm is uncertain over her own demand: to make things as simple as possible, we assume that she conjectures a linear demand defined by two parameters, a constant and the coefficient of its own price. She maintains the hypothesis that the distribution of the two parameters is a normal bivariate: the mean and the precision hyper-parameters of this 
distribution at date $t$ are as follows ${ }^{1}$ :

$$
\mathbf{m}_{i, t}=\left(\begin{array}{c}
\alpha_{i, t} \\
\beta_{i, t}
\end{array}\right) \quad \text { and } \quad \boldsymbol{\Gamma}_{i, t}=\left(\begin{array}{cc}
\gamma_{i,(1, t)} & \gamma_{i,(12, t)} \\
\gamma_{i,(12, t)} & \gamma_{i,(2, t)}
\end{array}\right)
$$

where $\alpha_{i, t}, \beta_{i, t}, \gamma_{i,(1, t)}$ and $\gamma_{i,(2, t)}$ are positive. Since the firm has surely no reason to conjecture any particular initial value for the correlation among the two mean hyper-parameters, we assume $\gamma_{i,(12,0)}=0$. For notational easiness we put $\gamma_{i,(1,0)} \equiv \gamma_{i 1}$ and $\gamma_{i,(2,0)} \equiv \gamma_{i 2}$. Since each firm correspond to a product (as in standard monopolistic competition) we use the $i$ index to define the firm, so we call $f_{i, t}(a, b) i$ firm's conjecture at date $t$.

As in the previous setting, the timing is the following: the firm announces the price before observing demand, hence uses its $(t-1)$-conjecture, formed observing demand at time $(t-1)$.

The firm chooses the price so as to maximize expected profit, $E_{i, t-1}\left(Q_{t} \mid p_{i, t}\right) \cdot\left(p_{i, t}-c_{i}\right)$. Therefore, from standard First Order Condition in monopoly, the price announced at date $t$ is $^{2}$

$$
p_{i, t}=\frac{\alpha_{i, t-1}}{2 \beta_{i, t-1}}+\frac{c_{i}}{2}=\frac{\alpha_{i, t-1}+c_{i} \beta_{i, t-1}}{2 \beta_{i, t-1}}
$$

and expect the following demand:

$$
Q_{i, t}^{e}\left(p_{i, t}\right)=\frac{\alpha_{i, t-1}-c_{i} \beta_{i, t-1}}{2}
$$

We neglect any capacity constraint, assuming the firm can meet all demand.

The updating process of her conjecture is following standard bayesian rules: the firm average the prior and the result of a recursive OLS estimation. We follow the same steps as in Bogliacino and Rampa 2008: define the row vector $\mathbf{x}_{t}^{\prime} \equiv\left[\begin{array}{ll}1 & -p_{t}\end{array}\right]$; given our assumptions, one has ${ }^{3}$ :

$$
\mathbf{m}_{i, t}=\left[\boldsymbol{\Gamma}_{i, t-1}+\mathbf{x}_{i, t} \mathbf{x}_{i, t}^{\prime}\right]^{-1}\left[\boldsymbol{\Gamma}_{i, t-1} \mathbf{m}_{i, t-1}+\mathbf{x}_{i, t} Q_{i, t}^{D}\right]
$$

and

$$
\boldsymbol{\Gamma}_{i, t}=\left[\boldsymbol{\Gamma}_{i, t-1}+\mathbf{x}_{i, t} \mathbf{x}_{i, t}^{\prime}\right]
$$

\footnotetext{
${ }^{1}$ We assume that the conditional distribution of $Q$ given $a, b$ and $p$ has known precision equal to 1 ; if this precision were different from 1 , the matrix of precision would be multiplied by its value. Things could be generalized, but this would be immaterial for our results, since firm's expected profit does not depend on precisions, given risk neutrality.

${ }^{2}$ It must of course be the case that $\alpha_{t} / \beta_{t}>c$, otherwise the firm would not find it profitable to sell anything.

${ }^{3}$ See De Groot 1970, chapter 11.
} 
By simple algebra, (3.3) can also be written as

$$
\begin{aligned}
\mathbf{m}_{i, t} & =\left[\boldsymbol{\Gamma}_{i, t-1}+\mathbf{x}_{i, t} \mathbf{x}_{i, t}^{\prime}\right]^{-1}\left[\left(\boldsymbol{\Gamma}_{i, t-1}+\mathbf{x}_{i, t} \mathbf{x}_{i, t}^{\prime}-\mathbf{x}_{i, t} \mathbf{x}_{i, t}^{\prime}\right) \mathbf{m}_{i, t-1}+\mathbf{x}_{i, t} Q_{i, t}^{D}\right]= \\
& =\left[\boldsymbol{\Gamma}_{i, t-1}+\mathbf{x}_{i, t} \mathbf{x}_{i, t}^{\prime}\right]^{-1}\left[\left(\boldsymbol{\Gamma}_{i, t-1}+\mathbf{x}_{i, t} \mathbf{x}_{i, t}^{\prime}\right) \mathbf{m}_{i, t-1}+\mathbf{x}_{i, t}\left(Q_{i, t}^{D}-\mathbf{x}_{i, t}^{\prime} \mathbf{m}_{i, t-1}\right)\right]= \\
& =\mathbf{m}_{i, t-1}+\left[\boldsymbol{\Gamma}_{i, t-1}+\mathbf{x}_{i, t} \mathbf{x}_{i, t}^{\prime}\right]^{-1}\left[\mathbf{x}_{i, t}\left(Q_{i, t}^{D}-\mathbf{x}_{i, t}^{\prime} \mathbf{m}_{i, t-1}\right)\right]
\end{aligned}
$$

In a nutshell, the above expression tells us that the new parameters are equal to the previous period ones, corrected for the prediction error (adjusted for the new precision).

\section{Equilibria: Main Results}

The system can be fully characterized in terms of the hyperparameters of the firms and the consumers. The risk aversion of the consumers make them sensible to all piece of information available: as time goes by, they increase their precisions raising their demand. For this reason, the system show path dependence and irreversibility and the relevant equilibrium concept is the steady state one, which in this setup has an interpretation in terms of the stability of the conjectures of the agents: it is in fact a conjectural equilibrium.

Define first $\boldsymbol{\mu}_{j, t}^{\prime}=\left[\begin{array}{lll}\mu_{j, 1, t} & \ldots & \mu_{j, n, t}\end{array}\right]$ and $\boldsymbol{\tau}_{j, t}^{\prime}=\left[\begin{array}{lll}\tau_{j, 1, t} & \ldots & \tau_{j, n, t}\end{array}\right]$, the vectors of a consumer's hyper-parameters at date $t$. Then extend it to all consumers: $\boldsymbol{\mu}_{t}^{\prime}=$ $\left[\begin{array}{lll}\mu_{1, t} & \ldots & \mu_{M, t}\end{array}\right]$ and $\boldsymbol{\tau}_{t}^{\prime}=\left[\begin{array}{lll}\tau_{1, t} & \ldots & \tau_{M, t}\end{array}\right]$. In order to do the same for firms, define $\gamma_{i, t}^{\prime}=\left[\begin{array}{llll}\gamma_{i,(1, t)} & \gamma_{i,(12, t)} & \gamma_{i,(12, t)} & \gamma_{i,(2, t)}\end{array}\right]$, the vectorization of matrix $\boldsymbol{\Gamma}_{i, t}$, and in the same spirit $\gamma_{t}^{\prime}=\left[\begin{array}{lll}\gamma_{1, t}^{\prime} & \cdots & \left.\gamma_{n, t}\right)^{\prime}\end{array}\right]$. Simmetrically we can define:

$$
\mathbf{m}_{\mathbf{t}}=\left[\mathbf{m}_{\mathbf{1}, \mathbf{t}}{ }^{\prime}, \mathbf{m}_{\mathbf{2}, \mathbf{t}}{ }^{\prime}, \ldots, \mathbf{m}_{\mathbf{n}, \mathbf{t}}\right]^{\prime}
$$

Finally, call $\mathbf{y}_{t}^{\prime}=\left[\begin{array}{llll}\boldsymbol{\mu}_{t}^{\prime} & \boldsymbol{\tau}_{t}^{\prime} & \mathbf{m}_{t}^{\prime} & \gamma_{t}^{\prime}\end{array}\right]$. We can compact all in the first order difference equations:

$$
\mathbf{y}_{t}=F\left(\mathbf{y}_{t-1}\right)
$$

which completely describes the diffusion dynamics. A conjectural equilibrium is a fixed point of this map ${ }^{4}$.

First of all, we remind the main results from the previous paper.

Proposition 1. Suppose that the quality is a normal process and suppose that demand for a good $i$ is positive at time $t$. Then, there exists positive probability of failure of the $i$ product at time $t+1$.

\footnotetext{
${ }^{4}$ All the proofs of the main results (Propositions 2 and 3 and Lemma 1 ) are available from the authors upon request, in a large mathematical Appendix.
} 
Proof. See Bogliacino and Rampa 2008.

The argument runs as follows: at every time $t$ we can build a complete ordering over the set of consumers in terms of a function of their hyperparameters: the higher the value, the higher the quality expected. If a signal is such biased as to drive below the threshold the most optimistic consumer (remember that $A<\exp \left(-\lambda_{i}\right)$ implies no purchase), then all demand is driven to zero. But then no information is made available to update and consumers are locked-in at zero demand ${ }^{5}$.

We now come to more general results. Once let the individual precisions to diverge, an equilibrium is such that the consumers' means converge and firms conjectures cross the true demands, in a way that the prediction error is zero. We can fix the ideas over the dynamics in expected value, i.e. with the signal always equal to the true quality. If this is true, using a Law of Large Number argument we can guarantee that consumers converge to the true quality levels. At the same time, defining:

$$
\mathbf{g}_{i, t}\left(\mathbf{m}_{\mathbf{t}}\right)=Q_{i, t}^{D}-\mathbf{x}_{i, t}^{\prime} \mathbf{m}_{i, t-1}
$$

we can write the following equilibrium conditions:

$$
\mathbf{g}_{t}\left(\mathbf{m}_{\mathbf{t}}\right)=\left[\mathbf{g}_{1, t}\left(\mathbf{m}_{\mathbf{t}}\right), \ldots, \mathbf{g}_{n, t}\left(\mathbf{m}_{\mathbf{t}}\right)\right]^{\prime}=0
$$

a set of $n$ equations in $2 n$ variables: the following Proposition 2 holds.

Proposition 2. There exists a n-dimensional equilibrium manifold in the space of agents' parameters.

A natural questions is then the stability of equilibria along the manifold. We should stress that we study stability at $t<\infty$, i.e. at what we called finite time. Being the system decomposable and given the convergence to one of the eigenvalues of the consumers' blocks of the jacobian, the stability along the manifold depends entirely on the firm's block, i.e. the impact of the firms' parameters (through prices) over the others. We can prove the following Proposition 3.

Proposition 3. Along the equilibrium manifold at finite time, the equilibria where conjectured demand is more elastic than the true one are locally unstable.

Proof. In Appendix.

The intuition for the result can be seen in the following Figure 1. One can see an equilibrium in $A$, where the firm maximize profits (given the conjecture) and there is no prediction error. From the definition of equilibrium, $\left(p^{*}, Q^{*}\right)$ are common to both the

\footnotetext{
${ }^{5}$ Another point we discussed in the previous paper, which is valid also here is the possibility of concave or logistic diffusion curve depending on the initial conditions, for a discussion see also Young 2007.
} 


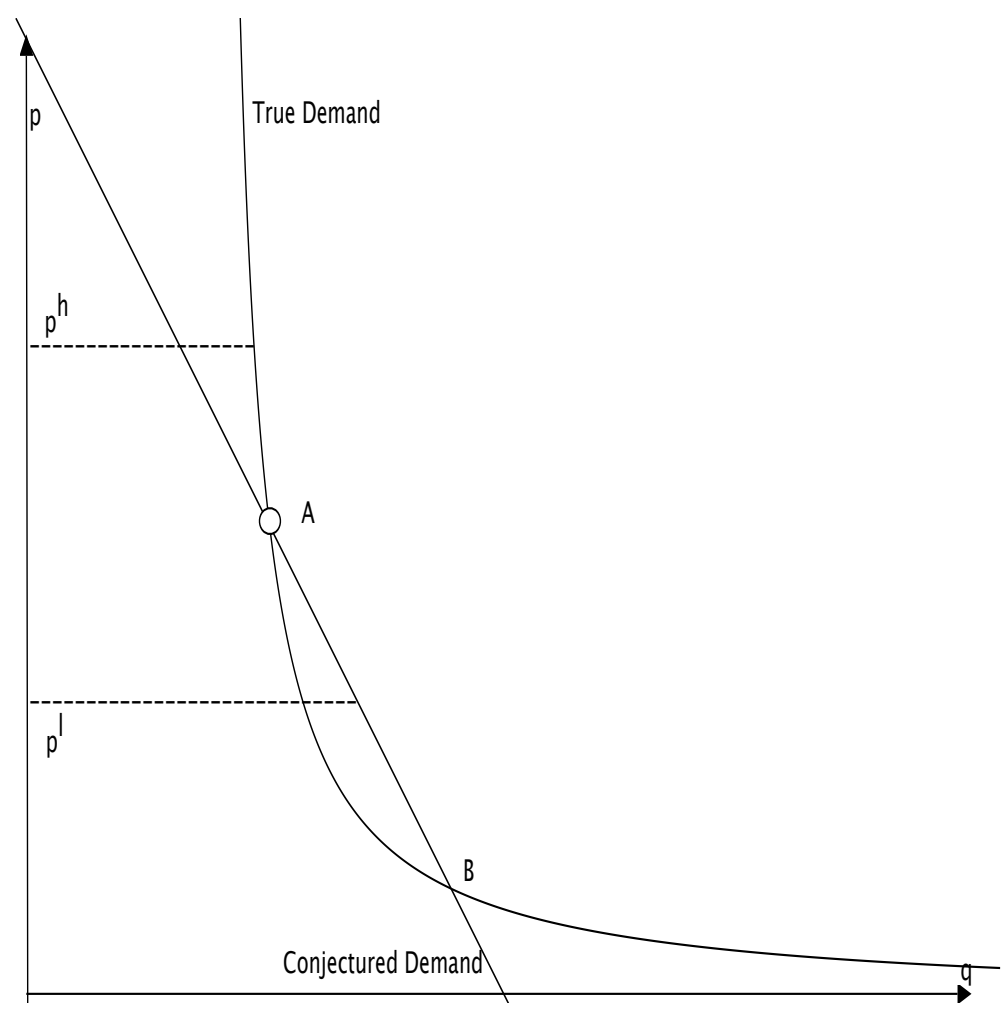

Figure 1: An equilibrium with conjectured demand more elastic than the true one.

true and the conjectured demand, so higher elasticity means that the derivative is higher (in absolute value) along the conjectured one, such as in $A$. At the opposite, a $B$-like equilibrium is one where the true demand is less rigid than the conjectured one.

We should look carefully at the (3.5): in presence of excess demand the firm's updated parameters grow, adjusting her conjecture towards a more rigid curve, the opposite for excess supply.

If we look at what is happening in a neighboorhood of $A$, we can see that a smaller price, such as $p^{l}$ generates excess supply, while a greater price, e.g. $p^{h}$, excess demand. It is then apparent that the firm will adjust moving away from the $A$ equilibrium. Similar reasoning for a $B$-like equilibrium shows that in this case there exists a basin of attraction.

This type of instability is local, in the sense that we are looking at a linear approssimation, thus in a neighborhood of an equilibrium, and it is caused by eigenvalues greater than one.

However, in finite times we can still see instability, of the oscillatory type (at least an eigenvalue less than -1) in the opposite case, but this property is smoothed by the passing of time and the instabiility is rapidly reabsorbed. 
Lemma 1. In equilibria where the conjectured demand is more rigid, there can exist oscillatory instability as long as $t<\infty$ and small, and with provided that firms' individual precisions are low.

Proof. In Appendix.

Finally we can add some minor results in terms of welfare. In Bogliacino and Rampa 2008 we analyze both welfare and stability along the manifold. In this case the $n$-dimensionality makes things complex: we have a problem of identifying the relevant direction along the manifold, and we cannot block $n-1$ firms, since moving one price obviously implies change in all expenditure shares. We leave this point for further research. However, this multiple goods setup allows us to analyze also the degree of diversification of the decentralized economy, although under some stricter assumptions.

Proposition 4. In equilibrium with endogenous number of firms (positive fixed cost of entry) and identical quality goods, there is over[respectively under]-diversification, if for the marginal firms (defined as that who fix the price at the lowest level) the true elasticity is greater [lower] than the conjectured one.

Proof. Let introduce a fixed cost of entry equal to $F$. In order to calculate the optimal degree of diversification, we need to fix price equal to marginal cost $p=c$, introduce lump sum taxation for an amount $n F / M$ for each consumer (reducing her income to $y-n F / M$ ) and maximize the indirect utility function in $n$.

The demand for identical quality goods is:

$$
Q_{D}=M(y-n F / M) \frac{p^{1 /(\delta-1)} f^{1 /(1-\delta)}}{n p^{\delta /(\delta-1) f^{1 /(1-\delta)}}}=M(y-n F / M) / n p
$$

the indirect utility function is (once replacing $p=c$ )

$$
n^{1-\delta} c^{-\delta} M^{\delta} f(y-n F / M)^{\delta}
$$

massimizzando in $n$ we get the following first order condition (which is also suficient due to strict concavity of the indirect utility function):

$$
n^{e}=(1-\delta) M y / F=\frac{M y}{F}\left(\epsilon_{T}\right)^{-1}
$$

The equilibrium condition with endogenous number of firms is a zero profit condition for the marginal firm, defined by the $\bar{p}$ price, where $\bar{p}=\min \left\{p_{i} \mid p_{i}=p_{i}^{*}\right\}$, given the equality of marginal cost and quality (and convergence of consumers' conjectures in equilibrium):

$$
(\bar{p}-c) Q=F \rightarrow \frac{\alpha-c \beta}{2 \beta} \frac{M y}{n p}=F
$$


by simple algebra

$$
n^{*}=\frac{M y}{F} \frac{\alpha-c \beta}{\alpha+c \beta}=\frac{M y}{F \epsilon_{C}}
$$

Over(under)-diversification is the case $n^{*}>n^{e}\left(n^{*}<n^{e}\right)$. Replacing for the two expressions above completes the proof.

The interpretation is fairly obvious: provided efficiency of the full information case, $\frac{\epsilon_{T}}{\epsilon_{C}}>1$ in equilibrium makes the firm less able to appropriate surplus, pushing entry. The opposite with $\frac{\epsilon_{T}}{\epsilon_{C}}<1$.

Thus, interestingly $s=1$ is not only stable, as in Bogliacino and Rampa 2008, it is also efficient in terms of diversification!

A Note: the result is partly dependent on the form of the utility function: in general the relation between the optimal degree of diversification and the one with perfect information depends on how the preferences affect the mark up, since it is related with the ability of the firm to appropriate the surplus (see Dixit and Stiglitz 1977, Tirole 1988, Bertoletti et al. 2008). In this case, the isoelastic case guarantees efficiency. However we can always say that the ratio among the two elasticity (the true and the conjectures) allows us to define over and under diversification w.r.t. the perfect information case, but in the general case we cannot say any longer that the perfect information one is also optimal.

\section{Conclusions}

This work characterizes a monopolistic competitive market where firms innovate introducing new products in an environment where they are uncertain about demand. At the same time, consumers are heterogenous in their expectations about quality. Through time there is interaction inter and intra market sides, which shapes the learning process and the final pattern observed.

This setup is fruitful since it allows for analysis of diffusion, failure and welfare. Moreover heterogeneity, interaction and analytical tractability coexist. We characterized analytically the set of equilibria (as one can expect, equilibrium it is not unique).

Further research includes the use of more sofisticated firms (oligopoly or conjectural variations models) and the characterization of the welfare properties along the manifold. Of course the model can be simulated to see different diffusion curves and how they vary from initial conditions. 


\section{References}

[1] Abrahamson E., Rosenkopf L. (1993), Institutional and competitive bandwagons: using mathematical modelling as a tool to explore innovation diffusion, Academy of Management Review, 18, 487-517

[2] Agliardi E. (1998), Positive Feedback Economies, London, Macmillan

[3] Amable B. (1992), Effets d'apprentissage, compétitivité hors-prix et croissance cumulative, Economie Appliquée, vol. 45(3), 5-31

[4] Aoki M. and H. Yoshikawa (2002), Demand Saturation-Creation amd Economic Growth, Journal od Economic Behavior and Organization, 48, 127-154

[5] Arthur B. W. (1994), Increasing Returns and Path Dependence in the Economy, Ann Arbor: University of Michigan Press

[6] Bertoletti, P., E. Fumagalli and C. Poletti (2008) On Price-Increasing Monopolistic Competition, working paper, University of Pavia

[7] Bogliacino, F. and G. Rampa (2008) Quality Risk Aversion, Conjectures, and New Product Diffusion, working paper, University of Genova

[8] Chatterjee R., Eliashberg J. (1990), The innovation diffusion process in a heterogeneous population: a micromodeling approach, Magament Science, 36, 1057-1079

[9] De Groot M. H. (1970), Optimal Statistical Decision, New York, McGraw Hill

[10] Dixit A. K. and J.E. Stiglitz (1977) Monopolistic competition and optimum product diversity, American Economic Review 67, 297-308.

[11] Feder G., O'Mara G.T. (1982), On information and innovation diffusion: a Bayesian approach, American Journal of Agricultural Economics, 64, 145-147

[12] Jensen R. (1982), Adoption and diffusion of an innovation of uncertain profitability, Journal of Economic Theory, 27, 182-193

[13] Lopez Pintado D., Watts D. J. (2006) Social Influence, Binary Decisions and Collective Dynamics, working paper

[14] Tirole, J. (1988) Industrial Organization. Cambridge: MIT Press

[15] Tsur Y., Sternberg M., Ochman E. (1990), Dynamic modelling of innovation process adoption with risk aversion and learning, Oxford Economic Papers, 42, 336-355

[16] Verbrugge R. (2000), Risk Aversion, Learning Spillovers, and Path-dependent Economic Growth, Economics Letters, 68, 197-202

[17] Young P. (2005) The Spread of Innovation through Social Learning, CSED working paper 12/2005

[18] Young P. (2007) Innovation Diffusion in Heterogeneous population, Economic Series Working Papers, 303, University of Oxford, Department of Economics 